Pacific

Journal of

Mathematics

\title{
ON THE FLAG CURVATURE OF A CLASS OF FINSLER METRICS \\ PRODUCED BY THE NAVIGATION PROBLEM
}

Libing HuANG AND XiaOHUAN Mo

Volume 277 No. 1

September 2015 


\title{
ON THE FLAG CURVATURE OF A CLASS OF FINSLER METRICS PRODUCED BY THE NAVIGATION PROBLEM
}

\author{
Libing HuANG AND XiaOHUAN Mo
}

\begin{abstract}
One of the important approaches in discussing Finsler geometry is the navigation problem. In this paper, we determine the flag curvature of a Finsler metric produced from any Finsler metric and any conformal field in terms of the navigation problem, and therefore we provide a unifying framework for the fundamental equations due to Bao, Robles, and Shen, Cheng and Shen, Foulon, and Mo and Huang.
\end{abstract}

\section{Introduction}

The navigation problem (or shortest time problem [Shen 2003]) was first studied by E. Zermelo [1931]. Bao, Robles, and Shen [Bao et al. 2004] classified Randers metrics of constant flag curvature via the navigation problem on Riemannian manifolds. Flag curvature is an important quantity in Finsler geometry because it takes the place of sectional curvature in the Riemannian case [Bao and Chern 1993]. The complete classification of Randers metrics of constant flag curvature, due to Bao, Robles, and Shen, is motivated by the following result [Bao et al. 2004; Chern and Shen 2005]:

Theorem. A Randers metric $F$ is of constant flag curvature $K=\lambda$ if and only if (i) $h$ has constant sectional curvature $\mu=\lambda+c^{2}$ and (ii) $V$ is a homothetic field of $h$ with dilation $c$, where $(h, V)$ is the navigation data of $F$.

Condition (ii) is equivalent to $F$ having constant $S$-curvature [Shen and Xing 2008; Xing 2005]. Recently, Cheng and Shen [2009] established a relationship between the flag curvature of $F$ and $h$ for a Randers metric $F$ of isotropic $S$-curvature (see also [Chern and Shen 2005]), generalizing the flag curvature nonincreasing equation of [Bao et al. 2004]. More generally, they obtained a relationship between the Riemann curvature of $F$ and $h$. Using this, they locally classified Randers metrics of scalar flag curvature with isotropic $S$-curvature [Cheng and Shen 2009;

Xiaohuan Mo is the corresponding author. Huang was supported by the National Natural Science Foundation of China 11301283. Mo was supported by the National Natural Science Foundation of China 11371032.

MSC2010: 58E20.

Keywords: Finsler metric, flag curvature, conformal field, navigation problem. 
Chern and Shen 2005; Shen 2004]. Mo [2008] gave a global classification for these metrics on compact manifolds by using a formula of Cheng and Shen [2009]. It is worth mentioning the recent result by Xing [Shen and Xing 2008] that a Randers metric $F$ is of isotropic $S$-curvature $S=(n+1) c(x) F$ if and only if $V$ is conformal with respect to $h$. The theorems of Cheng and Shen [2003] and Mo generalize results previously only known in the case of locally projectively flat Randers metrics with isotropic $S$-curvature. Recall that all locally projectively flat Finsler metrics are of scalar curvature [Chern and Shen 2005, Proposition 6.1.3].

Recall that a vector field $V$ on a Finsler manifold $(M, F)$ is called conformal with dilation $c(x)$ if its flow $\Phi_{t}$ satisfies

$$
F\left(\Phi_{t}(x), \Phi_{t *}(y)\right)=e^{2 \sigma_{t}(x)} F(x, y), \quad \forall x \in M, y \in T_{x} M,
$$

where $c(x)=\left[d \sigma_{t}(x) / d t\right]_{t=0}$ [Shen and Xia 2012; Huang and Mo 2013]. In particular, $V$ is called a homothetic field if $c$ is constant, and $V$ is called a Killing field if $c=0$ [Huang and Mo 2011; Mo and Hang 2007].

At the 2004 International Conference on Riemann-Finsler Geometry at Nankai University, P. Foulon announced that if $F$ is a Finsler metric and $V$ is a Killing field, then $F$ and $\widetilde{F}$ have the same flag curvature. Mo and Huang [2007] studied the navigation problem for any Finsler metric $F$ and any homothetic field (for instance, the Funk metric on a strongly convex domain) in the spirit of the flag curvature nonincreasing equation of Bao, Robles, and Shen and the announcement of P. Foulon. They showed that for a homothetic field, the navigation representation satisfies the flag curvature nonincreasing equation. In particular, the navigation problem has the flag curvature preserving property for a Killing field. Applying this result, $\mathrm{Hu}$ and Deng [2012] established a principle to classify homogeneous Randers spaces with (almost) isotropic S-curvature and positive flag curvature, and then they gave a complete classification of these homogeneous Randers spaces.

In this paper, we provide a unifying framework for [Bao et al. 2004; Cheng and Shen 2009; Mo and Hang 2007]. We study the Finsler metric $\widetilde{F}$ produced from any Finsler metric $F$ and any conformal field $V$ in terms of the shortest time problem and give the relation between the flag curvatures of $F$ and $\widetilde{F}$. Precisely we show the following:

Theorem 1.1. Let $F=F(x, y)$ be a Finsler metric on a manifold $M$ with Cartan torsion $A$ and $V$ be a vector field on $M$ with $F\left(x, V_{x}\right)<1$. Let $\widetilde{F}=\widetilde{F}(x, y)$ denote the Finsler metric on $M$ defined in (2-2). Suppose that $V$ is conformal with dilation $c(x)$. Then the flag curvatures of $\widetilde{F}$ and $F$ are related by $K_{\widetilde{F}}(y, y \wedge u)-\left[3 \frac{y^{i} c_{x^{i}}}{\widetilde{F}(x, y)}-c^{2}+2 V(c)\right]=K_{F}(\tilde{y}, \tilde{y} \wedge u)-2 \frac{A_{(x,[\tilde{y}])}(u, \nabla c, u)}{h_{(x,[\tilde{y}])}(u, u)}$, where $\tilde{y}=y+F(x, \tilde{y}) V$ and $h$ is the angular metric of $F$. 
For the definition of a conformal field $V$ with dilation $c(x)$, see Section 2. In Theorem 1.1, we denote the partial derivative $\partial c / \partial x^{i}$ by $c_{x^{i}}$. The case where $F$ is a Riemannian manifold implies a formula of Cheng and Shen [2009], whilst $V$ is homothetic implies the curvature nonincreasing equation of Mo and Huang [2007]. In particular, if $\widetilde{F}$ has constant flag curvature and is of Randers type, our formula has been obtained by Bao, Robles, and Shen [2004].

Our approach to proving Theorem 1.1 is partially in the contact geometry [Blair 2002]. Recall that a Finsler metric is Riemannian if and only if its Cartan torsion vanishes [Chern and Shen 2005].

As an application of Theorem 1.1, we determine the flag curvature of a Finsler metric produced by a generalized Poincaré metric and its nonhomothetic conformal field via the navigation problem (see Section 5).

Finally, we should point out that very recently [Shen and Xia 2012; Xia 2013] established the relationship between the flag curvatures of $\widetilde{F}$ and $F$, where $F$ is a Randers metric with some special curvature properties and $\widetilde{F}$ is produced from $(F, V)$ via the navigation problem, where $V$ is a conformal field.

\section{Preliminaries}

Let $(M, F)$ be a Finsler manifold with Hilbert form $\omega$. Let $S M$ be the projective sphere bundle of $M$, obtained from $T M$ by identifying nonzero vectors which differ from each other by a positive multiplicative factor. It is easy to verify that

$$
\omega \wedge(d \omega)^{n-1} \neq 0, \quad n=\operatorname{dim} M .
$$

That is, $\omega$ defines a contact structure on $S M$ [Chern 1996]. Hence there is a unique vector field $X$ on $S M$ that satisfies $\omega(X)=1$ and $X\lrcorner(d \omega)=0$. This vector field $X$ is known as the Reeb vector field [Blair 2002; Bryant 2002; Huang and Mo 2011].

Every vector $y \in T_{x} M \backslash\{0\}$ uniquely determines a covector $p \in T_{x}^{*} M \backslash\{0\}$ by

$$
p(u):=\left.\frac{1}{2} \frac{d}{d t}\left(F^{2}(x, y+t u)\right)\right|_{t=0}, \quad u \in T_{x} M .
$$

The resulting map $L_{x}^{F}: y \in T_{x} M \rightarrow p \in T_{x}^{*} M$ is called the Legendre transformation at $x$.

Define a nonnegative scalar function $H=H(x, p)$ by

$$
H(x, p):=\max _{y \in T_{x} M \backslash\{0\}} \frac{p(y)}{F(x, y)} .
$$

The function $H$ is $C^{\infty}$ on $T^{*} M \backslash\{0\}$ and $H_{x}:=\left.H\right|_{T_{x}^{*} M}$ is a Minkowski norm on $T_{x}^{*} M$ for $x \in M$. Such a function is called a Cartan metric [Miron et al. 2001; Mo and Hang 2007] (or co-Finsler metric [Shen 2004; 2002]). The pair $(M, H)$ is called a Cartan manifold. 
Every covector $p \in T_{x}^{*} M \backslash\{0\}$ uniquely determines a vector $y \in T_{x} M \backslash\{0\}$ by

$$
q(y):=\left.\frac{1}{2} \frac{d}{d t}\left(H^{2}(x, p+t q)\right)\right|_{t=0}, \quad q \in T_{x}^{*} M .
$$

The resulting map $L_{x}^{F *}: p \in T_{x}^{*} M \rightarrow y \in T_{x} M$ is called the inverse Legendre transformation at $x$. Indeed $L_{x}^{F}$ and $L_{x}^{F *}$ are inverses of each other. Moreover, they preserve the Minkowski norms $H(x, p)=F\left(x, L_{x}^{F *} p\right)$.

Recently, one of the important approaches in discussing Finsler metrics is the (Zermelo) navigation problem [Bao et al. 2004; Hu and Deng 2012; Huang and Mo 2011; Shen 2003; Zermelo 1931; Xia 2013]. The main technique of the navigation problem is described as follows. Given a Finsler metric $F$ and a vector field $V$ with $F\left(x, V_{x}\right)<1$, define a new Finsler metric $\widetilde{F}$ by

$$
F\left(x, \frac{y}{\widetilde{F}(x, y)}+V_{x}\right)=1, \quad \forall x \in M, y \in T_{x} M .
$$

A (local) flow (or local one-parameter group) on a manifold $M$ is a map $\Phi:(-\epsilon, \epsilon) \times M \rightarrow M$, also denoted by $\Phi_{t}:=\Phi(t, \cdot)$, satisfying

- $\Phi_{0}=\mathrm{id}: M \rightarrow M$;

- $\Phi_{s} \circ \Phi_{t}=\Phi_{s+t}$ for any $s, t \in(-\epsilon, \epsilon)$ with $s+t \in(-\epsilon, \epsilon)$.

Hence, the lift of a flow $\Phi_{t}$ on $M$ is a flow $\hat{\Phi}_{t}$ on $T^{*} M$,

$$
\hat{\Phi}_{t}(x, p):=\left(\Phi_{t}(x),\left(\Phi_{t}^{*}\right)^{-1}(p)\right) .
$$

By the relationship between vector fields and flows, (2-3) induces a natural way a lift of a vector field $V$ on $M$ to a vector field $X_{V}^{*}$ on $T^{*} M$.

A vector field $V$ on a Finsler manifold $(M, F)$ is called conformal with dilation $c(x)$ if its flow $\Phi_{t}$ satisfies

$$
F\left(\Phi_{t}(x), \Phi_{t *}(y)\right)=e^{2 \sigma_{t}(x)} F(x, y), \quad \forall x \in M, y \in T_{x} M,
$$

where $c(x)=\left[d \sigma_{t}(x) / d t\right]_{t=0}$ [Shen and Xia 2012]. In particular, $V$ is called a homothetic field if $c$ is constant.

Similarly, a vector field $V$ on a Cartan manifold $(M, H)$ is called conformal with dilation $c(x)$ if its flow $\Phi_{t}$ is a conformal transformation on $(M, H)$, i.e.,

$$
H\left(\Phi_{t}(x),\left(\Phi_{t}^{*}\right)^{-1}(p)\right)=e^{-2 \sigma_{t}(x)} H(x, p), \quad \forall x \in M, p \in T_{x}^{*} M,
$$

where $c(x)=\left[d \sigma_{t}(x) / d t\right]_{t=0}$.

Lemma 2.1. Let $V$ be a conformal field on a Finsler manifold $(M, F)$ with dilation $c(x)$ and $H$ its Cartan metric defined by (2-1). Then $V$ is a conformal field of $H$ with dilation $c(x)$. 
Proof. By using (2-1) and (2-4) we have

$$
\begin{aligned}
H\left(\Phi_{t}(x),\left(\Phi_{t}^{*}\right)^{-1}(p)\right) & =\max _{\tilde{y} \in T_{\Phi_{t}(x)} M \backslash\{0\}} \frac{\left[\left(\Phi_{t}^{*}\right)^{-1}(p)\right](\tilde{y})}{F\left(\Phi_{t}(x), \tilde{y}\right)} \\
& =\max _{\tilde{y} \in T_{\Phi_{t}(x)} M \backslash\{0\}} \frac{p\left(\left(\Phi_{t *}\right)^{-1}(\tilde{y})\right)}{F\left(\Phi_{t}(x), \tilde{y}\right)} \\
& =\max _{y \in T_{x} M \backslash\{0\}} \frac{p(y)}{F\left(\Phi_{t}(x), \Phi_{t *}(y)\right)} \\
& =\max _{y \in T_{x} M \backslash\{0\}} \frac{p(y)}{e^{2 \sigma_{t}(x)} F(x, y)} \\
& =e^{-2 \sigma_{t}(x)} \max _{y \in T_{x} M \backslash\{0\}} \frac{p(y)}{F(x, y)}=e^{-2 \sigma_{t}(x)} H(x, p),
\end{aligned}
$$

where $y:=\left(\Phi_{t *}\right)^{-1}(\tilde{y})$. The lemma follows.

The Hilbert form $\omega^{b}$ of the co-Finsler metric $H$ is given by

$$
\omega^{b}=\frac{p}{H}
$$

[Mo and Hang 2007]. Let $S^{*} M$ be the cosphere bundle of $M$ and $\pi: S^{*} M \rightarrow M$ the natural projection. We call Ker $\pi_{*}$ the vertical distribution of $S^{*} M$, denoted by $V S^{*} M$.

Lemma 2.2. For an arbitrary function $f \in C^{\infty}\left(S^{*} M\right)$, there is a unique vector field $X_{f}$ on $S^{*} M$ satisfying

$$
\left.\omega^{\mathrm{b}}\left(X_{f}\right)=f, \quad X_{f}\right\lrcorner\left(d \omega^{\mathrm{b}}\right)=-d f+X^{\mathrm{b}}(f) \omega^{\mathrm{b}} .
$$

This vector field $X_{f}$ is called the Reeb field associated with $f$.

Proof. The Hilbert form $\omega^{\mathrm{b}}$ defines a contact structure on $S^{*} M$. By using [Blair 2002, Theorem 4.4], there exists an almost contact metric structure $\left(\Phi, X^{\mathrm{b}}, \omega^{\mathrm{b}}, g\right)$ such that $g(X, \Phi Y)=d \omega^{\mathrm{b}}(X, Y)$. A direct computation tells us that the second equation of (2-7) is equivalent to $\mathcal{L}_{X_{f}} \omega^{\mathrm{b}}=X^{\mathrm{b}}(f) \omega^{\mathrm{b}}$. Together with [loc. cit., Theorem 5.7], we have $X_{f}=-\Phi D f+f X^{b}$, where $g(D f, Y)=Y(f)$.

Remark. (i) It is easy to see that $X_{1}=X^{\mathrm{b}}$ is known as the Reeb vector field.

(ii) Let $\left\{e_{\alpha}, X^{\mathrm{b}}, e_{\bar{\alpha}}\right\}$ be a locally orthonormal frame on $S^{*} M$ such that $e_{\alpha} \in H S^{*} M$ (see (2-10) below) and $e_{\bar{\alpha}} \in V S^{*} M$. By using (2-7), we have

$$
X_{f}=f X^{\mathrm{b}}+\Sigma_{\alpha} e_{\bar{\alpha}}(f) e_{\alpha}-\Sigma_{\alpha} e_{\alpha}(f) e_{\bar{\alpha}} .
$$

By the definition of $V S^{*} M$, we have $e_{\bar{\alpha}}(f)=0$ for $f \in C^{\infty}(M)$. It follows that

$$
Y_{f}:=X_{f}-f X^{b}=-\Phi D f \in V S^{*} M \text { for } f \in C^{\infty}(M) \text {. }
$$


(iii) Note that the $d \omega^{b}$ adopted here differs from that of D. E. Blair [2002], where $d \omega^{\mathrm{b}}$ is defined by

$$
d \omega^{\mathrm{b}}(X, Y)=\frac{1}{2}\left(X\left(\omega^{\mathrm{b}}(Y)\right)-Y\left(\omega^{\mathrm{b}}(X)\right)-\omega^{\mathrm{b}}([X, Y])\right) .
$$

In the same work, $X_{f}$ is called an infinitesimal contact transformation.

Let $F$ be a Finsler metric and $\widetilde{F}$ denote the Finsler metric defined in (2-2). With the help of the inverse Legendre transformation at $x$, we obtain co-Finsler metrics $H(x, p)$ and $\tilde{H}(x, p)$ respectively. Then $H$ and $\widetilde{H}$ are related by

$$
\tilde{H}(x, p)=H(x, p)-p(V)
$$

[Mo and Hang 2007]. Furthermore the Hilbert form $\tilde{\omega}^{\text {b }}$ of the co-Finsler metric $\tilde{H}$ satisfies $\tilde{\omega}^{b}=p / \widetilde{H}$. Taking this together with (2-6), we obtain $\operatorname{Ker} \omega^{b}=\operatorname{Ker} \tilde{\omega}^{b}$. The vertical endomorphism $\mathcal{V}^{b}$ is characterized by

$$
\mathcal{V}^{b}(v)=0, \quad \mathcal{V}^{b}\left(X^{b}\right)=0, \quad \mathcal{V}^{b}\left[X^{b}, v\right]=-v, \quad \forall v \in V S^{*} M .
$$

The horizontal endomorphism $\mathcal{H}^{b}$ is given by

$$
\mathcal{H}^{b}(v)=-\left[X^{b}, v\right]-\frac{1}{2} \mathcal{V}^{b}\left[X^{b},\left[X^{b}, v\right]\right], \quad \mathcal{H}^{b}\left(X^{b}\right)=0, \quad \mathcal{H}^{b}\left(\mathcal{H}^{b}(v)\right)=0
$$

for $v \in V S^{*} M$. The horizontal distribution of $S^{*} M$ is defined by

$$
H S^{*} M=\mathcal{H}^{b}\left(V S^{*} M\right) .
$$

It is easy to see that

$$
T S^{*} M=H S^{*} M \oplus V S^{*} M \oplus \operatorname{Span}\left\{X^{\mathrm{b}}\right\}=\operatorname{Ker} \omega^{\mathrm{b}} \oplus \operatorname{Span}\left\{X^{\mathrm{b}}\right\} .
$$

We denote the projection to $V S^{*} M$ (resp. $H S^{*} M$ ) by $P_{\mathcal{V}}^{b}:=\mathcal{V}^{b} \circ \mathcal{H}^{b}$ (resp. $P_{\mathcal{H}}^{b}:=$ $\mathcal{H}^{b} \circ \mathcal{V}^{b}$ ). Define the Riemann tensor of $\mathcal{R}^{b}$ by

$$
\mathcal{R}^{b}(v)=\mathcal{V}^{b} \circ \mathcal{H}^{b}\left[X^{b}, \mathcal{H}^{b}(v)\right], \quad v \in V S^{*} M .
$$

Then the flag curvature $K^{b}$ is given by

$$
K^{b}(v)=\frac{h^{b}\left(\mathcal{R}^{b}(v), v\right)}{h^{b}(v, v)}, \quad v \in V S^{*} M \backslash\{0\},
$$

where $h^{\mathrm{b}}$ is the angular metric on $V S^{*} M$ which satisfies

$$
h^{\mathrm{b}}(v, v)=d \omega^{\mathrm{b}}\left(\left[X^{\mathrm{b}}, u\right], v\right)=d \omega^{\mathrm{b}}\left(u, \mathcal{H}^{\mathrm{b}}(v)\right) .
$$

The Cartan torsion $A^{\text {b }}$ is characterized by $2 A^{\mathrm{b}}(u, v, w)=u\left(d \omega^{\mathrm{b}}\left(\left[X^{\mathrm{b}}, v\right], w\right)+d \omega^{\mathrm{b}}\left(\left[u,\left[X^{\mathrm{b}}, v\right]\right], w\right)+d \omega^{\mathrm{b}}\left(\left[u,\left[X^{\mathrm{b}}, w\right]\right], v\right)\right)$ 
for $u, v, w \in V S^{*} M$. We require the following result in Lemma 3.5, the proof of which is omitted.

Lemma 2.3. There is a unique affine connection $\nabla: V S^{*} M \times V S^{*} M \rightarrow V S^{*} M$ satisfying

$$
\nabla_{u} v=\mathcal{V}^{b}\left[u, \mathcal{H}^{\mathrm{b}}(v)\right], \quad \nabla_{u} v-\nabla_{v} u=[u, v], \quad\left(\nabla_{u} h^{\mathrm{b}}\right)(v, w)=2 A^{\mathrm{b}}(u, v, w)
$$

for $u, v, w \in V S^{*} M$.

The following lemma will be used in Section 4.

Lemma 2.4 [Mo and Hang 2007]. Assume that Cartan metrics $H$ and $\tilde{H}$ are related by (2-9). Then vertical endomorphisms $\mathcal{V}^{b}$ and $\tilde{\mathcal{V}}^{b}$ are related by $\mathcal{V}^{b}=$ $\tilde{\mathcal{V}}^{b}-\tilde{\mathcal{V}}^{b}\left(X_{V}^{*}\right) \otimes \omega^{b}$, where $X_{V}^{*}$ is the left of $V$ on $T^{*} M$.

\section{Conformal transformations}

In this section, we establish some properties for a conformal transformation on a Cartan manifold required in next section. For the definition of conformal transformation, see (2-5) above.

Lemma 3.1. Let $\varphi$ be a conformal transformation on a Cartan manifold $(M, H)$, i.e., $\hat{\varphi}^{*} H=e^{-2 \sigma(x)} H$, where $\hat{\varphi}(x, p)=\left(\varphi(x),\left(\varphi^{*}\right)^{-1}(p)\right)$. Then

$$
\hat{\varphi}_{*} X^{b}=X_{e^{2 \sigma(x)}},
$$

where $X^{b}$ denotes the Reeb field of $H$.

Proof. By (2-5) and (2-6), we have

$$
\hat{\varphi}^{*} \omega^{b}=e^{2 \sigma(x)} \omega^{b} .
$$

Hence $\hat{\varphi}: S^{*} M \rightarrow S^{*} M$ is a contact transformation [Blair 2002]. It follows that

$$
\omega^{b}\left(\hat{\varphi}_{*} X^{b}\right)=\left(\hat{\varphi}^{*} \omega^{b}\right) X^{b}=e^{2 \sigma(x)} \omega^{b}\left(X^{b}\right)=e^{2 \sigma(x)}
$$

and

$$
\begin{aligned}
\left.\hat{\varphi}_{*} X^{\mathrm{b}}\right\lrcorner\left(d \omega^{\mathrm{b}}\right) & \left.=X^{\mathrm{b}}\right\lrcorner\left(\hat{\varphi}^{*} d \omega^{\mathrm{b}}\right) \\
& \left.=X^{\mathrm{b}}\right\lrcorner\left[d\left(\hat{\varphi}^{*} \omega^{\mathrm{b}}\right)\right] \\
& \left.=X^{\mathrm{b}}\right\lrcorner\left[d\left(e^{2 \sigma(x)} \omega^{\mathrm{b}}\right)\right] \\
& \left.=X^{\mathrm{b}}\right\lrcorner\left[d e^{2 \sigma(x)} \wedge \omega^{\mathrm{b}}+e^{2 \sigma(x)} d \omega^{\mathrm{b}}\right] \\
& \left.=d e^{2 \sigma(x)}\left(X^{\mathrm{b}}\right) \omega^{\mathrm{b}}-\omega^{\mathrm{b}}\left(X^{\mathrm{b}}\right) d e^{2 \sigma(x)}+e^{2 \sigma(x)} X^{\mathrm{b}}\right\lrcorner\left(d \omega^{\mathrm{b}}\right) \\
& =-d e^{2 \sigma(x)}+X^{\mathrm{b}}\left(e^{2 \sigma(x)}\right) \omega^{\mathrm{b}} .
\end{aligned}
$$

The lemma follows from the uniqueness of the Reeb field associated with $e^{2 \sigma(x)}$. 
Proposition 3.2. Let $\varphi$ be a conformal transformation on a Cartan manifold $(M, H)$, i.e., $\hat{\varphi}^{*} H=e^{-2 \sigma(x)} H$. Then $\hat{\varphi}_{*} X^{b}=e^{2 \sigma(x)}\left(X^{b}+2 Y_{\sigma(x)}\right)$, where $Y_{\sigma(x)}$ is defined in (2-8).

Proof. By virtue of (2-8), we conclude that

$$
Y_{e^{2 \sigma(x)}}=-\Phi D e^{2 \sigma(x)}=2 e^{2 \sigma(x)}(-\Phi D \sigma(x))=2 e^{2 \sigma(x)} Y_{\sigma(x)} .
$$

It follows that

$$
\begin{aligned}
\hat{\varphi}_{*} X^{\mathrm{b}} & =X_{e^{2 \sigma(x)}} \\
& =Y_{e^{2 \sigma(x)}+e^{2 \sigma(x)} X^{\mathrm{b}}} \\
& =2 e^{2 \sigma(x)} Y_{\sigma(x)}+e^{2 \sigma(x)} X^{\mathrm{b}}=e^{2 \sigma(x)}\left(X^{\mathrm{b}}+2 Y_{\sigma(x)}\right) .
\end{aligned}
$$

Lemma 3.3. For a conformal transformation $\varphi$ on a Cartan manifold $(M, H)$, we have

$$
\hat{\varphi}_{*} \circ \mathcal{V}^{b}=e^{-2 \sigma(x)} \mathcal{V}^{b} \circ \hat{\varphi}_{*} .
$$

Proof. For $v \in V S^{*} M$ and $\hat{\varphi}_{*} v \in V S^{*} M$, it follows that

$$
\hat{\varphi}_{*} \circ \mathcal{V}^{b}(v)=0=e^{-2 \sigma(x)} \mathcal{V}^{b} \circ \hat{\varphi}_{*}(v) \text {. }
$$

Similarly, from (i) we have $\hat{\varphi}_{*} \circ \mathcal{V}^{\mathrm{b}}\left(X^{\mathrm{b}}\right)=e^{-2 \sigma(x)} \mathcal{V}^{\mathrm{b}} \circ \hat{\varphi}_{*}\left(X^{\mathrm{b}}\right)$. For $u \in H S^{*} M$, we write $u=\mathcal{H}^{\mathrm{b}}(v)$, where $v \in V S^{*} M$. Then

$$
\begin{aligned}
\hat{\varphi}_{*} \circ \mathcal{V}^{b}(u) & =\hat{\varphi}_{*} \circ \mathcal{V}^{b}\left(-\left[X^{b}, v\right]-\frac{1}{2} \mathcal{V}^{b}\left[X^{b},\left[X^{b}, v\right]\right]\right) \\
& =-\hat{\varphi}_{*} \circ \mathcal{V}^{b}\left[X^{b}, v\right]-\frac{1}{2} \hat{\varphi}_{*} \circ \mathcal{V}^{b} \circ \mathcal{V}^{b}\left[X^{b},\left[X^{b}, v\right]\right]=\hat{\varphi}_{*} v,
\end{aligned}
$$

and

$$
\begin{aligned}
e^{-2 \sigma(x)} \mathcal{V}^{\mathrm{b}} \circ \hat{\varphi}_{*}(u) & =e^{-2 \sigma(x)} \mathcal{V}^{\mathrm{b}} \circ \hat{\varphi}_{*}\left(\mathcal{H}^{\mathrm{b}}(v)\right) \\
& =e^{-2 \sigma(x)} \mathcal{V}^{\mathrm{b}} \circ \hat{\varphi}_{*}\left(-\left[X^{\mathrm{b}}, v\right]-\frac{1}{2} \mathcal{V}^{\mathrm{b}}\left[X^{\mathrm{b}},\left[X^{\mathrm{b}}, v\right]\right]\right) \\
& =-e^{-2 \sigma(x)} \mathcal{V}^{\mathrm{b}}\left[\hat{\varphi}_{*} X^{\mathrm{b}}, \hat{\varphi}_{*} v\right] \\
& =-e^{-2 \sigma(x)} \mathcal{V}^{\mathrm{b}}\left[e^{2 \sigma(x)} X^{\mathrm{b}}, \hat{\varphi}_{*} v\right] \\
& =-e^{-2 \sigma(x)} e^{2 \sigma(x)} \mathcal{V}^{\mathrm{b}}\left[X^{\mathrm{b}}, \hat{\varphi}_{*} v\right]=\hat{\varphi}_{*} v
\end{aligned}
$$

Lemma 3.4. Write $X^{b}(f)=\dot{f}$ for an arbitrary function $f \in C^{\infty}(M)$. Then

Proof. Simple calculations give

$$
\left[X^{b}, X_{f}\right]=X_{\dot{f}}
$$

$$
\left.\omega^{\mathrm{b}}\left(\left[X^{\mathrm{b}}, X_{f}\right]\right)=\dot{f}, \quad\left[X^{\mathrm{b}}, X_{f}\right]\right\lrcorner\left(d \omega^{\mathrm{b}}\right)=-d \dot{f}+\ddot{f} \omega^{\mathrm{b}} .
$$

The lemma now follows from the uniqueness of the Reeb field associated with $\dot{f}$. 
Lemma 3.5. If $f \in C^{\infty}(M)$ and $v \in V S^{*} M$, then

$$
\mathcal{V}^{b}\left[X_{\dot{f}}, v\right]=-2 A^{b}\left(Y_{f}, v\right),
$$

where $h^{\mathrm{b}}\left(A^{\mathrm{b}}\left(Y_{f}, v\right), u\right):=A^{\mathrm{b}}\left(v, Y_{f}, u\right)$.

Proof. By (2-8) and Lemma 3.4, we have

$$
\begin{aligned}
\mathcal{V}^{b} X_{\dot{f}} & =\mathcal{V}^{\mathrm{b}}\left[X^{\mathrm{b}}, X_{f}\right] \\
& =\mathcal{V}^{\mathrm{b}}\left[X^{\mathrm{b}}, Y_{f}+f X^{\mathrm{b}}\right] \\
& =\mathcal{V}^{\mathrm{b}}\left[X^{\mathrm{b}}, Y_{f}\right]+\mathcal{V}^{\mathrm{b}}\left[X^{\mathrm{b}}(f) X^{\mathrm{b}}\right] \\
& =-Y_{f}+X^{\mathrm{b}}(f) \mathcal{V}^{\mathrm{b}}\left(X^{\mathrm{b}}\right)=-Y_{f} .
\end{aligned}
$$

Note that $\left[P_{\mathcal{V}}^{b} X_{\dot{f}}, v\right] \in V S^{*} M$. It follows that

$$
\mathcal{V}^{b}\left[P_{\mathcal{V}} X_{\dot{f}}, v\right]=0
$$

Together with (2-7) and (3-3), we obtain

$$
\begin{aligned}
\mathcal{V}^{b}\left[X_{\dot{f}}, v\right] & =\mathcal{V}^{b}\left[\dot{f} X^{b}+P_{\mathcal{H}}^{b} X_{\dot{f}}+P_{\mathcal{V}}^{b} X_{\dot{f}}, v\right] \\
& =\mathcal{V}^{b}\left[\dot{f} X^{b}+\mathcal{H}^{b} \circ \mathcal{V}^{b} X_{\dot{f}}, v\right] \\
& =\mathcal{V}^{b}\left[\dot{f} X^{b}-\mathcal{H}^{b} Y_{f}, v\right]=\mathcal{V}^{b}\left[\dot{f} X^{b}, v\right]-\mathcal{V}^{b}\left[\mathcal{H}^{b} Y_{f}, v\right] .
\end{aligned}
$$

On the other hand,

$$
\mathcal{V}^{b}\left[\dot{f} X^{b}, v\right]=-v(\dot{f}) \mathcal{V}^{b}\left(X^{b}\right)+\dot{f} \mathcal{V}^{b}\left[X^{b}, v\right]=-\dot{f} v .
$$

Plugging this into (3-5) yields $\mathcal{V}^{b}\left[X_{\dot{f}}, v\right]=-\dot{f} v+\mathcal{V}^{b}\left[v, \mathcal{H}^{b} Y_{f}\right]$. It follows that

$$
\begin{aligned}
h^{b}\left(\mathcal{V}^{b}\left[X_{\dot{f}}, v\right], u\right) & =-\dot{f} h^{b}(v, u)+h^{b}\left(\mathcal{V}^{b}\left[v, \mathcal{H}^{b} Y_{f}\right], u\right) \\
& =-\dot{f} h^{b}(v, u)+h^{b}\left(\nabla_{v} Y_{f}, u\right) .
\end{aligned}
$$

By Lemma 2.3, we have

$$
\begin{aligned}
h^{\mathrm{b}}\left(\nabla_{v} Y_{f}, u\right) & =-\left(\nabla_{v} h^{\mathrm{b}}\right)\left(Y_{f}, u\right)-h^{\mathrm{b}}\left(Y_{f}, \nabla_{v} u\right)+v\left(h^{\mathrm{b}}\left(Y_{f}, u\right)\right) \\
& =-2 A^{\mathrm{b}}\left(v, Y_{f}, u\right)-h^{\mathrm{b}}\left(Y_{f}, \nabla_{v} u\right)+v\left(h^{\mathrm{b}}\left(Y_{f}, u\right)\right) .
\end{aligned}
$$

By a straightforward computation, one obtains

$$
h^{\mathrm{b}}\left(Y_{f}, v\right)=-\mathcal{H}^{\mathrm{b}}(v)(f)=-d f\left(\mathcal{H}^{\mathrm{b}}(v)\right), \quad v \in V S^{*} M .
$$

It follows that

$$
h^{\mathrm{b}}\left(Y_{f}, \nabla_{v} u\right)=-\left(P_{\mathcal{H}}^{\mathrm{b}}\left[v, \mathcal{H}^{\mathrm{b}}(u)\right]\right)(f)
$$

and

$$
v\left(h^{\mathrm{b}}\left(Y_{f}, u\right)\right)=-\left(P_{\mathcal{H}}^{\mathrm{b}}\left[v, \mathcal{H}^{\mathrm{b}}(u)\right]\right)(f)+\dot{f} h^{\mathrm{b}}(u, v),
$$


where we have used $h^{\mathrm{b}}(u, v)=-\omega^{\mathrm{b}}\left[u, \mathcal{H}^{\mathrm{b}}(v)\right]$. Substituting (3-8) and (3-9) into (3-7) and then combining it with (3-6), we have (3-2).

Proposition 3.6. For a conformal transformation $\varphi$ on a Cartan manifold $(M, H)$, we have

$$
\hat{\varphi}_{*} \mathcal{H}^{\mathrm{b}}(v)=e^{2 \sigma(x)}\left[\mathcal{H}^{\mathrm{b}}\left(\hat{\varphi}_{*} v\right)+2 \dot{\sigma} \hat{\varphi}_{*} v-2 A^{\mathrm{b}}\left(Y_{\sigma}, \hat{\varphi}_{*} v\right)\right] .
$$

Proof. By Lemma 3.3, we have

$$
\begin{aligned}
\hat{\varphi}_{*} \mathcal{H}^{b}(v) & =-\hat{\varphi}_{*}\left[X^{b}, v\right]-\frac{1}{2} \hat{\varphi}_{*} \circ \mathcal{V}^{b}\left[X^{b},\left[X^{b}, v\right]\right] \\
& =-\left[\hat{\varphi}_{*} X^{b}, \hat{\varphi}_{*} v\right]-\frac{1}{2} e^{-2 \sigma(x)} \mathcal{V}^{b} \circ \hat{\varphi}_{*}\left[X^{\mathrm{b}},\left[X^{\mathrm{b}}, v\right]\right] \\
& =-\left[e^{2 \sigma(x)}\left(X^{\mathrm{b}}+2 Y_{\sigma(x)}\right), \hat{\varphi}_{*} v\right]+(I),
\end{aligned}
$$

where

$$
\begin{aligned}
&(I)=-\frac{1}{2} e^{-2 \sigma(x)} \mathcal{V}^{\mathrm{b}}\left[\hat{\varphi}_{*} X^{\mathrm{b}}, \hat{\varphi}_{*}\left[X^{\mathrm{b}}, v\right]\right] \\
&=-\frac{1}{2} e^{-2 \sigma(x)} \mathcal{V}^{\mathrm{b}}\left[e^{2 \sigma(x)}\left(X^{\mathrm{b}}+2 Y_{\sigma(x)}\right), \hat{\varphi}_{*}\left[X^{\mathrm{b}}, v\right]\right] \\
&=-\frac{1}{2} e^{-2 \sigma(x)} \mathcal{V}^{\mathrm{b}}\left(-\hat{\varphi}_{*}\left[X^{\mathrm{b}}, v\right]\left(e^{2 \sigma(x)}\right)\left(X^{\mathrm{b}}+2 Y_{\sigma(x)}\right)\right)\left.\quad-\frac{1}{2} \mathcal{V}^{\mathrm{b}}\left[X^{\mathrm{b}}+2 Y_{\sigma(x)},\left[\hat{\varphi}_{*} X^{\mathrm{b}}, \hat{\varphi}_{*} v\right]\right]\right] \\
&=-\frac{1}{2} e^{-2 \sigma(x)}\left(-\hat{\varphi}_{*}\left[X^{\mathrm{b}}, v\right]\left(e^{2 \sigma(x)}\right) \mathcal{V}^{\mathrm{b}}\left(X^{\mathrm{b}}+2 Y_{\sigma(x)}\right)\right) \\
&\left.\quad-\frac{1}{2} \mathcal{V}^{\mathrm{b}}\left[X^{\mathrm{b}}+2 Y_{\sigma(x)}, e^{2 \sigma(x)}\left[X^{\mathrm{b}}+2 Y_{\sigma(x)}, \hat{\varphi}_{*} v\right]\right]\right] \\
&=-\frac{1}{2}\left((I I)+e^{2 \sigma(x)} \mathcal{V}^{b}\left[X^{\mathrm{b}}+2 Y_{\sigma(x)},\left[X^{\mathrm{b}}+2 Y_{\sigma(x)}, \hat{\varphi}_{*} v\right]\right]\right),
\end{aligned}
$$

and

$$
\begin{aligned}
(I I) & =\mathcal{V}^{\mathrm{b}}\left(X^{\mathrm{b}}+2 Y_{\sigma(x)}\right)\left(e^{2 \sigma(x)}\right)\left[X^{\mathrm{b}}+2 Y_{\sigma(x)}, \hat{\varphi}_{*} v\right] \\
& =X^{\mathrm{b}}\left(e^{2 \sigma(x)}\right)\left(\mathcal{V}^{\mathrm{b}}\left[X^{\mathrm{b}}, \hat{\varphi}_{*} v\right]+2 \mathcal{V}^{\mathrm{b}}\left[Y_{\sigma(x)}, \hat{\varphi}_{*} v\right]\right)=-X^{\mathrm{b}}\left(e^{2 \sigma(x)}\right) \hat{\varphi}_{*} v .
\end{aligned}
$$

Plugging this into (3-12) and combining with (3-11), we obtain

$$
\hat{\varphi}_{*} \mathcal{H}^{\mathrm{b}}(v)=e^{2 \sigma(x)}\left(\mathcal{H}^{\mathrm{b}}\left(\hat{\varphi}_{*} v\right)-\left[Y_{\sigma(x)}, \hat{\varphi}_{*} v\right]+X^{\mathrm{b}}(v) \hat{\varphi}_{*} v-\mathcal{V}^{\mathrm{b}}\left[Y_{\sigma(x)},\left[X^{\mathrm{b}}, \hat{\varphi}_{*} v\right]\right]\right) .
$$

By using the Jacobi identity and Lemma 3.4, we have

$$
\begin{aligned}
-\mathcal{V}^{b}\left[Y_{\sigma(x)},\left[X^{b}, \hat{\varphi}_{*} v\right]\right] & =\mathcal{V}^{b}\left[X^{b},\left[\hat{\varphi}_{*} v, Y_{\sigma(x)}\right]\right]-\mathcal{V}^{b}\left[\hat{\varphi}_{*} v,\left[X^{b}, Y_{\sigma(x)}\right]\right] \\
& =-\left[\hat{\varphi}_{*} v, Y_{\sigma(x)}\right]-\mathcal{V}^{b}\left[\hat{\varphi}_{*} v, X_{\dot{\sigma}}\right] .
\end{aligned}
$$

Plugging this into (3-13) and using Lemma 3.5, we get (3-10).

\section{Conformal navigation problems}

We call the navigation problem (2-2) conformal if $V$ is a conformal field. In this section, we explore some properties of conformal navigation problems and prove Theorem 1.1. 
Lemma 4.1. Let $V$ be a conformal field on a Cartan manifold $(M, H)$ with dilation $c(x)$. Let $\tilde{H}$ be the Cartan metric given in (2-9). Then for $v \in V S^{*} M$

$$
\mathcal{H}^{b}(v)=\widetilde{\mathcal{H}}^{b}(v)-c v,
$$

where $\mathcal{H}^{b}$ (resp. $\left.\tilde{\mathcal{H}}^{b}\right)$ is the horizontal endomorphism of $H$ (resp. $\tilde{H}$ ).

Proof. By [Mo and Hang 2007, Lemma 4.10], we have

$$
\left[X^{\mathrm{b}}, v\right] \in \operatorname{Ker} \omega^{\mathrm{b}}=H S^{*} M \oplus V S^{*} M, \quad\left[X^{\mathrm{b}},\left[X^{\mathrm{b}}, v\right]\right] \in \operatorname{Ker} \omega^{\mathrm{b}} .
$$

Together with Lemma 2.4 we get

$$
-\mathcal{H}^{b}(v)=\left[X^{b}, v\right]+\frac{1}{2} \tilde{\mathcal{V}}^{b}\left[X^{b},\left[X^{b}, v\right]\right] .
$$

According to [loc. cit., Lemma 6.2], the Reeb fields of $X^{b}$ and $\tilde{X}^{b}$ satisfy

$$
X^{b}=\tilde{X}^{b}+X_{V}^{*}
$$

where

$$
X_{V}^{*}=v^{i} \frac{\partial}{\partial x^{i}}-p_{j} \frac{\partial v^{j}}{\partial x^{i}} \frac{\partial}{\partial p_{i}},
$$

with $V=v^{i}\left(\partial / \partial x^{i}\right)$. It follows that

$$
\begin{aligned}
\tilde{\mathcal{V}}^{b}\left[X^{b},\left[X^{\mathrm{b}}, v\right]\right] & =\tilde{\mathcal{V}}^{b}\left[\tilde{X}^{b},\left[X^{\mathrm{b}}, v\right]\right]+\tilde{\mathcal{V}}^{\mathrm{b}}\left[X_{V}^{*},\left[X^{\mathrm{b}}, v\right]\right] \\
& =\tilde{\mathcal{V}}^{\mathrm{b}}\left[\tilde{X}^{\mathrm{b}},\left[\tilde{X}^{\mathrm{b}}, v\right]\right]+\tilde{\mathcal{V}}^{\mathrm{b}}\left[\tilde{X}^{\mathrm{b}},\left[X_{V}^{*}, v\right]\right]+\tilde{\mathcal{V}}^{\mathrm{b}}\left[X_{V}^{*},\left[X^{\mathrm{b}}, v\right]\right] .
\end{aligned}
$$

Let $\hat{\varphi}_{t}$ be flow of $X_{V}^{*}$. Then $\left(\hat{\varphi}_{t}\right)_{*} v$ is vertical for $v \in V S^{*} M$. Hence,

$$
\left[X_{V}^{*}, v\right]:=\lim _{t \rightarrow 0} \frac{v-\left(\hat{\varphi}_{t}\right)_{*} v}{t}
$$

is also vertical. It follows that

$$
\tilde{\mathcal{V}}^{b}\left[\tilde{X}^{b},\left[X_{V}^{*}, v\right]\right]=-\left[X_{V}^{*}, v\right] .
$$

By using the Jacobi identity, we have

$$
\left[X_{V}^{*},\left[X^{\mathrm{b}}, v\right]\right]=-\left[X^{\mathrm{b}},\left[v, X_{V}^{*}\right]\right]-\left[v,\left[X_{V}^{*}, X^{\mathrm{b}}\right]\right] .
$$

Now we assume that $V$ is a conformal field of Cartan metric $H$ with dilation $c(x)$; that is, the flow $\varphi_{t}$ of $V$ satisfies

$$
\hat{\varphi}_{t}^{*} H=e^{-2 \sigma_{t}(x)} H, \quad c(x)=\left[\frac{d \sigma_{t}(x)}{d t}\right]_{t=0} .
$$

Differentiating the first of these equations with respect to $t$ at $t=0$ yields 


$$
\begin{aligned}
-2 c(x) H & =\left.\frac{\partial}{\partial t}\left(e^{-2 \sigma_{t}(x)} H\right)\right|_{t=0} \\
& =\left.\frac{\partial}{\partial t}\left(\hat{\varphi}_{t}^{*} H\right)\right|_{t=0} \\
& =\left.\frac{\partial}{\partial t}\left(H \circ \hat{\varphi}_{t}\right)\right|_{t=0} \\
& =\left.\frac{\partial \hat{\varphi}_{t}}{\partial t}\right|_{t=0} H=X_{V}^{*}(H) .
\end{aligned}
$$

Recall that $V S^{*} M=\operatorname{Ker} \pi_{*}=\left\{v \in T S M \mid v(f)=0, \forall f \in C^{\infty}(M) \subset C^{\infty}\left(S^{*} M\right)\right\}$. Together with (4-2), we have

$$
\left[v, 2 c X^{b}\right]=v(2 c) X^{b}-2 c\left[X^{b}, v\right]=-2 c\left[X^{b}, v\right] \in \operatorname{Ker} \omega^{b} .
$$

Note that the vertical distribution is involutive. We obtain

$$
\tilde{\mathcal{V}}^{b}\left[v, \frac{\partial c}{\partial x^{i}} H \frac{\partial}{\partial p_{i}}\right]=0 .
$$

A direct calculation (see [Huang and Mo 2011, Lemma 3.2]) gives the formula

$$
\left[X^{\mathrm{b}}, X_{V}^{*}\right]=2 c X^{\mathrm{b}}-2 \frac{\partial c}{\partial x^{i}} H \frac{\partial}{\partial p_{i}} .
$$

By Lemma 2.4, we obtain

$$
\tilde{\mathcal{V}}^{b}\left[v,\left[X_{V}^{*}, X^{b}\right]\right]=2 \tilde{\mathcal{V}}^{b}\left[v, \frac{\partial c}{\partial x^{i}} H \frac{\partial}{\partial p_{i}}\right]-\tilde{\mathcal{V}}^{b}\left[v, 2 c X^{b}\right]=-2 c v .
$$

Together with (4-6) and (4-8), we have

$$
\begin{aligned}
\tilde{\mathcal{V}}^{b}\left[X_{V}^{*},\left[X^{b}, v\right]\right] & =-\tilde{\mathcal{V}}^{b}\left[X^{b},\left[v, X_{V}^{*}\right]\right]-\tilde{\mathcal{V}}^{b}\left[v,\left[X_{V}^{*}, X^{b}\right]\right] \\
& =-\mathcal{V}^{b}\left[X^{b},\left[v, X_{V}^{*}\right]\right]+2 c v=\left[v, X_{V}^{*}\right]+2 c v .
\end{aligned}
$$

Plugging this and (4-7) into (4-5) yields

$$
\begin{aligned}
\tilde{\mathcal{V}}^{b}\left[X^{b},\left[X^{b}, v\right]\right] & =\tilde{\mathcal{V}}^{b}\left[\tilde{X}^{b},\left[\tilde{X}^{b}, v\right]\right]-\left[X_{V}^{*}, v\right]+\left[v, X_{V}^{*}\right]+2 c v \\
& =\tilde{\mathcal{V}}^{b}\left[\tilde{X}^{b},\left[\tilde{X}^{b}, v\right]\right]-2\left[X_{V}^{*}, v\right]+2 c v .
\end{aligned}
$$

Substituting this into (4-3) and using (4-4), we deduce that

$$
\begin{aligned}
-\mathcal{H}^{b}(v) & =\left[X^{b}, v\right]+\frac{1}{2}\left(\tilde{\mathcal{V}}^{b}\left[\tilde{X}^{b},\left[\tilde{X}^{b}, v\right]\right]-2\left[X_{V}^{*}, v\right]+2 c v\right) \\
& =\left[\tilde{X}^{b}, v\right]+\frac{1}{2} \tilde{\mathcal{V}}^{b}\left[\tilde{X}^{b},\left[\tilde{X}^{b}, v\right]\right]+c v=-\tilde{\mathcal{H}}^{b}(v)+c v .
\end{aligned}
$$

This gives (4-1). 
Lemma 4.2. Let $V$ be a conformal field on a Cartan manifold $(M, H)$ with dilation $c(x)$. Let $\widetilde{H}$ be the Cartan metric given in (2-9). Then on $H S^{*} M \oplus V S^{*} M$, we have $P_{\widetilde{\mathcal{V}}}^{\mathrm{b}}=P_{\mathcal{V}}^{\mathrm{b}}-c \tilde{\mathcal{V}}^{\mathrm{b}}=P_{\mathcal{V}}^{\mathrm{b}}-c \mathcal{V}^{\mathrm{b}}$, where $P_{\mathcal{V}}^{\mathrm{b}}\left(\right.$ resp. $\left.P_{\widetilde{\mathcal{V}}}^{\mathrm{b}}\right)$ is the projection of $H($ resp. $\widetilde{H})$.

Proof. The second equality follows from Lemma 2.4. For $v \in V S^{*} M$,

$$
\tilde{\mathcal{V}}^{b}(v)=0, \quad P_{\widetilde{\mathcal{V}}}^{b}(v)=P_{\mathcal{V}}^{b}
$$

It follows that

$$
P_{\widetilde{\mathcal{V}}}^{b}(v)=\left(P_{\mathcal{V}}^{b}-c \tilde{\mathcal{V}}^{b}\right)(v), \quad \forall v \in V S^{*} M
$$

For $u \in H S^{*} M$, we write $u \in \mathcal{H}^{b}(v)$, where $v \in V S^{*} M$. By the definition of $\widetilde{\mathcal{H}}^{b}$ and Lemma 4.1, we obtain

$$
\begin{aligned}
P_{\tilde{\mathcal{V}}}^{b}(u)+c \tilde{\mathcal{V}}^{b}(u) & =\tilde{\mathcal{V}}^{b} \circ \tilde{\mathcal{H}}^{b}\left(\mathcal{H}^{b}(v)\right)+c \tilde{\mathcal{V}}^{b}\left(\mathcal{H}^{b}(v)\right) \\
& =\tilde{\mathcal{V}}^{b} \circ \tilde{\mathcal{H}}^{b}\left(\tilde{\mathcal{H}}^{b}(v)-c v\right)+c \tilde{\mathcal{V}}^{b}\left(\tilde{\mathcal{H}}^{b}(v)-c v\right) \\
& =-c \tilde{\mathcal{V}}^{b} \circ \tilde{\mathcal{H}}^{b}(v)+\tilde{\mathcal{V}}^{b} \circ \tilde{\mathcal{H}}^{b}(v)-c^{2} \tilde{\mathcal{V}}^{b}(v) \\
& =0=P_{\mathcal{V}}^{b}(u) .
\end{aligned}
$$

Proposition 4.3. Let $V$ be a conformal field of $H$ with dilation $c(x)$. Then

$$
\left[X_{V}^{*}, \mathcal{H}^{b}(v)\right]=-2 c \mathcal{H}^{b}(v)+\mathcal{H}^{b}\left[X_{V}^{*}, v\right]-2 \dot{c} v+2 A^{b}\left(Y_{c}, v\right) .
$$

Proof. By using Proposition 3.6, we have

$$
\begin{aligned}
{\left[X_{V}^{*}, \mathcal{H}^{\mathrm{b}}(v)\right] } & =-\left.\frac{d}{d t}\right|_{t=0} \hat{\varphi}_{t *} \mathcal{H}^{\mathrm{b}}(v) \\
& =-\left.\frac{d}{d t}\right|_{t=0}\left(e^{2 \sigma_{t}(x)}\left[\mathcal{H}^{\mathrm{b}}\left(\hat{\varphi}_{t *} v\right)+2 \dot{\sigma}_{t} \hat{\varphi}_{t *} v-2 A^{\mathrm{b}}\left(Y_{\sigma_{t}}, \hat{\varphi}_{t *} v\right)\right]\right),
\end{aligned}
$$

where $\varphi_{t}$ is the flow of $V$. By direct calculations, we have

$$
\begin{gathered}
-\left.\frac{d}{d t}\right|_{t=0} \mathcal{H}^{\mathrm{b}}\left(\hat{\varphi}_{t *} v\right)=\mathcal{H}^{\mathrm{b}}\left[X_{V}^{*}, v\right], \quad-\left.\frac{d}{d t}\right|_{t=0} A^{\mathrm{b}}\left(Y_{\sigma_{t}}, \hat{\varphi}_{t *} v\right)=A^{\mathrm{b}}\left(Y_{c}, v\right), \\
-\left.\frac{d}{d t}\right|_{t=0}\left(\dot{\sigma}_{t} \hat{\varphi}_{t *} v\right)=\left.\frac{d \dot{\sigma}_{t}}{d t}\right|_{t=0} \hat{\varphi}_{0 *} v+\left.\dot{\sigma}_{t}\right|_{t=0} \frac{d}{d t} \hat{\varphi}_{t *} v=X^{\mathrm{b}}(c) v=\dot{c} v .
\end{gathered}
$$

Plugging them into (4-11), we have (4-10).

Proposition 4.4. Let $V$ be a conformal field on a Cartan manifold $(M, H)$ with dilation $c(x)$. Let $\tilde{H}$ be the Cartan metric given in (2-9). Then

$$
\tilde{\mathcal{R}}^{\mathrm{b}}(v)=\mathcal{R}^{\mathrm{b}}(v)+\left[3 \tilde{X}^{\mathrm{b}}(c)-c^{2}+2 X_{V}^{*}(c)\right] v-2 A^{\mathrm{b}}\left(Y_{c}, v\right),
$$

where $\mathcal{R}^{b}$ (resp. $\left.\tilde{\mathcal{R}}^{b}\right)$ is the Riemann tensor of $H$ (resp. $\left.\tilde{H}\right)$. 
Proof. From [Mo and Hang 2007, Lemma 4.9], we have

$$
P_{\mathcal{V}}^{b}\left[X^{b}, v\right]=\mathcal{V}^{b}\left[X^{b}, \mathcal{H}^{b}(v)\right], \quad v \in V S^{*} M .
$$

By (2-11), (4-1) and (4-4),

$$
\begin{aligned}
\tilde{\mathcal{R}}^{b}(v) & =P_{\widetilde{\mathcal{V}}}^{b}\left[\tilde{X}^{\mathrm{b}}, \tilde{\mathcal{H}}^{\mathrm{b}}(v)\right] \\
& =P_{\widetilde{\mathcal{V}}}^{\mathrm{b}}\left[\tilde{X}^{\mathrm{b}}, \mathcal{H}^{\mathrm{b}}(v)+c v\right] \\
& =P_{\widetilde{\mathcal{V}}}^{\mathrm{b}}\left[\tilde{X}^{\mathrm{b}}, \mathcal{H}^{\mathrm{b}}(v)\right]+P_{\widetilde{\mathcal{V}}}^{\mathrm{b}}\left[\tilde{X}^{\mathrm{b}}, c v\right] \\
& =P_{\widetilde{\mathcal{V}}}^{\mathrm{b}}\left[X^{\mathrm{b}}-X_{V}^{*}, \mathcal{H}^{\mathrm{b}}(v)\right]+P_{\widetilde{\mathcal{V}}}^{\mathrm{b}}\left(\tilde{X}^{\mathrm{b}}(c) v+c\left[\tilde{X}^{\mathrm{b}}, v\right]\right) \\
& =(I)-P_{\widetilde{\mathcal{V}}}^{\mathrm{b}}\left[X_{V}^{*}, \mathcal{H}^{\mathrm{b}}(v)\right]+\tilde{X}^{\mathrm{b}}(c) v,
\end{aligned}
$$

where

$$
\begin{aligned}
(I):= & P_{\widetilde{\mathcal{V}}}^{\mathrm{b}}\left[X^{\mathrm{b}}, \mathcal{H}^{\mathrm{b}}(v)\right]+c P_{\widetilde{\mathcal{V}}}^{\mathrm{b}}\left[\tilde{X}^{\mathrm{b}}, v\right] \\
= & \left(P_{\mathcal{V}}^{\mathrm{b}}-c \mathcal{V}^{\mathrm{b}}\right)\left[X^{\mathrm{b}}, \mathcal{H}^{\mathrm{b}}(v)\right]+c\left(P_{\mathcal{V}}^{\mathrm{b}}-c \mathcal{V}^{\mathrm{b}}\right)\left[X^{\mathrm{b}}-X_{V}^{*}, v\right] \\
= & P_{\mathcal{V}}^{b}\left[X^{\mathrm{b}}, \mathcal{H}^{\mathrm{b}}(v)\right]-c \mathcal{V}^{\mathrm{b}}\left[X^{\mathrm{b}}, \mathcal{H}^{\mathrm{b}}(v)\right]+c P_{\mathcal{V}}^{b}\left[X^{\mathrm{b}}, v\right] \\
& \quad-c^{2} \mathcal{V}^{b}\left[X^{\mathrm{b}}, v\right]-c P_{\mathcal{V}}^{b}\left[X_{V}^{*}, v\right]+c^{2} \mathcal{V}^{b}\left[X_{V}^{*}, v\right] \\
& =\mathcal{R}^{\mathrm{b}}(v)+c^{2} v-c\left[X_{V}^{*}, v\right],
\end{aligned}
$$

where we have used (4-13). It follows that

$$
\tilde{\mathcal{R}}^{b}(v)=\mathcal{R}^{b}(v)-P_{\widetilde{\mathcal{V}}}^{\mathrm{b}}\left[X_{V}^{*}, \mathcal{H}^{\mathrm{b}}(v)\right]-c\left[X_{V}^{*}, v\right]+\left[\tilde{X}^{\mathrm{b}}(c)+c^{2}\right] v .
$$

From (4-4), we have

$$
\tilde{X}^{b}(c)=\dot{c}-X_{V}^{*}(c) .
$$

By using (4-11) and Lemma 4.2, we obtain

$$
\begin{aligned}
P_{\widetilde{\mathcal{V}}}^{\mathrm{b}}\left[X_{V}^{*}, \mathcal{H}^{\mathrm{b}}(v)\right]= & 2 c^{2} P_{\mathcal{V}}^{\mathrm{b}}-c P_{\mathcal{V}}^{\mathrm{b}}\left[X_{V}^{*}, v\right]-2 \dot{c} P_{\mathcal{V}}^{\mathrm{b}} v \\
& \quad+2 c \dot{c} \mathcal{V}^{\mathrm{b}}(v)+2 P_{\mathcal{V}}^{\mathrm{b}} A^{\mathrm{b}}\left(Y_{c}, v\right)-2 c \mathcal{V}^{\mathrm{b}} A^{\mathrm{b}}\left(Y_{c}, v\right) \\
= & 2 c^{2} v-c\left[X_{V}^{*}, v\right]-2 \dot{c} v+2 A^{\mathrm{b}}\left(Y_{c}, v\right) .
\end{aligned}
$$

Plugging this and (4-15) into (4-14) yields (4-12).

Proposition 4.5. Let $V$ be a conformal field on a Cartan manifold $(M, H)$ with dilation $c(x)$. Let $\tilde{H}$ be the Cartan metric given in (2-9). Then

$$
\tilde{K}^{b}(v)-\left[3 \tilde{X}^{b}(c)-c^{2}+2 V(c)\right]=K^{b}(v)-2 \frac{A^{b}\left(v, Y_{c}, v\right)}{h^{b}(v, v)},
$$

where $K^{\mathrm{b}}\left(\right.$ resp. $\left.\tilde{K}^{\mathrm{b}}\right)$ is the flag curvature of $H($ resp. $\tilde{H})$. 
Proof. By [Mo and Hang 2007, Lemma 6.2], we have $h^{b}\left(v_{1}, v_{2}\right)=(\tilde{H} / H) \tilde{h}^{b}\left(v_{1}, v_{2}\right)$. Together with (4-12) and (2-12), this yields

$$
\tilde{K}^{b}(v)=K^{b}(v)+3 \tilde{X}^{b}(c)-c^{2}+2 X_{V}^{*}(c)-2 \frac{A^{b}\left(v, Y_{c}, v\right)}{h^{b}(v, v)} .
$$

On the other hand,

$$
X_{V}^{*}(c)=\left(v^{i} \frac{\partial}{\partial x^{i}}-p_{j} \frac{\partial v^{j}}{\partial x^{i}} \frac{\partial}{\partial p_{i}}\right) c(x)=v^{i} \frac{\partial c}{\partial x^{i}}=V(c) .
$$

Together with (4-17), we have (4-16).

Proof of Theorem 1.1. Let $F$ be a Finsler metric with flag curvature $K$, Cartan torsion $A$ and angular metric $h$. Let $V$ be a conformal field on $M$ with $F\left(x, V_{x}\right)<1$. Let $\widetilde{F}$ be the Finsler metric given in (2-2) with flag curvature $\widetilde{K}$. Then their Cartan metrics are related by (2-9). From Lemma 2.1, we obtain that $V$ is a conformal field of $H$ with dilation $c(x)$. Hence $K$ and $\widetilde{K}$ satisfy (4-16). By (2-8), we have $A^{\mathrm{b}}\left(v, Y_{c}, v\right)=-A^{\mathrm{b}}(v, \Phi D c, v)$. Plugging this into (4-16) yields

$$
\begin{aligned}
{\left[\tilde{K}^{b}(v)\right]_{(x,[p])}-\left[3 \tilde{X}^{b}(c)-c^{2}+2 V(c)\right]_{(x,[p])} } & \\
& =\left[K^{b}(v)\right]_{(x,[p])}+2 \frac{A^{b}(v, \Phi D c, v)_{(x,[p])}}{h^{b}(v, v)_{(x,[p])}} .
\end{aligned}
$$

Pulling back to the sphere bundle, we have

$$
[\tilde{K}(u)]_{(x,[y])}-\left[3 \frac{y^{i} c_{x^{i}}}{\widetilde{F}}-c^{2}+2 V(c)\right]=[K(u)]_{(x,[\tilde{y}])}-2 \frac{A(u, \nabla c, u)_{(x,[\tilde{y}])}}{h(u, u)_{(x,[\tilde{y}])}},
$$

where $u:=\left(L_{x}^{F *}\right)_{*} v, \nabla c:=\left(L_{x}^{F *}\right)_{*} \Phi D c$ and where we have used $\partial \tilde{H} / \partial p_{i}=y^{i} / \tilde{F}$. By [Mo and Hang 2007, Lemma 3.9], we get the desired result.

Remark. (i) The reader should note that the navigation problem adopted here differs from that of [Shen and Xia 2012; Shen 2003], where the navigation problem is defined by $F(x, y / \widetilde{F}(x, y)-V)=1$; i.e., the $\widetilde{F}$ that we define with $(F, V)$ is precisely the $\widetilde{F}$ that Shen defines with $(F,-V)$.

(ii) We have two special cases of Theorem 1.1:

(1) If $V$ is homothetic, i.e., its dilation $c(x)$ is constant, then $\nabla c=0$ and our formula is reduced to that of Mo and Huang [2007].

(2) If $F$ is Riemannian and has sectional curvature $K=K(x)$, then our formula is reduced to that of Cheng and Shen [2009] (see also [Chern and Shen 2005]). 


\section{An example}

In this section, we determine the flag curvature of a nontrivial example using Theorem 1.1.

Consider the case $\operatorname{dim} M=2$; so $x=\left(x^{1}, x^{2}\right)$ and $y=\left(y^{1}, y^{2}\right)$. In order to avoid the excessive use of parentheses, we shall abbreviate $x^{1}, x^{2}$ as $s, t$ and $y^{1}, y^{2}$ as $p, q$. Let

$$
M:=\left\{(s, t) \in \mathbb{R}^{2} \mid t>1\right\} .
$$

Define $F: T M \rightarrow \mathbb{R}$ by

$$
F(s, t ; p, q):=\frac{1}{t} \Phi(p, q),
$$

where

$$
\Phi(p, q):=\left(p^{4}+2 \epsilon p^{2} q^{2}+q^{4}\right)^{1 / 4}, \quad \epsilon \in(0,3),
$$

is a Minkowski norm on $\mathbb{R}^{2}$ (see [Shen 2001, Example 1.1.3]) and $F$ is a Finsler metric on $M$.

For the Finsler surface $(M, F)$, its Gaussian curvature $K$ takes the place of the flag curvature in general case. A direct calculation shows that the Gaussian curvature of $F$ is given by

$$
K_{F}(s, t ; p, q)=\frac{[\Phi(p, q)]^{2} Q(p, q)}{[\Delta(p, q)]^{4}},
$$

where

$$
\begin{aligned}
Q(p, q):=\epsilon & \left(2 \epsilon^{2}-3\right) p^{14}+\left(17 \epsilon^{4}-42 \epsilon^{3}+18\right) p^{12} q^{2}+\epsilon\left(8 \epsilon^{4}-50 \epsilon^{2}+21\right) p^{10} q^{4} \\
+ & \left(9 \epsilon^{6}-89 \epsilon^{4}+81 \epsilon^{2}-36\right) p^{8} q^{6}-5 \epsilon\left(5 \epsilon^{4}-4 \epsilon^{2}+6\right) p^{6} q^{8} \\
& +\epsilon^{2}\left(5 \epsilon^{4}-5 \epsilon^{2}-21\right) p^{4} q^{10}+\epsilon^{3}\left(5 \epsilon^{2}-12\right) p^{2} q^{12}-\epsilon^{4} q^{14}
\end{aligned}
$$

and

$$
\Delta(p, q):=\epsilon p^{4}+\left(3-\epsilon^{2}\right) p^{2} q^{2}+\epsilon q^{4} .
$$

We denote the determinant of the fundamental tensor by $g$. Then

$$
g=\frac{\Delta(p, q)}{t^{4}[\Phi(p, q)]^{4}},
$$

where we have used (5-1), (5-2) and (5-5). The Cartan form $\eta$ is given by

$$
\eta=\left(F \frac{\partial}{\partial y^{j}} \log \sqrt{g}\right) d x^{j} .
$$


Then the main scalar $I$ of $F$ is given by

$$
\begin{aligned}
I(x, y) & =\eta\left(e_{1}\right) \\
& =\frac{-1}{\sqrt{g}}\left(\left(\frac{\partial}{\partial p} \log \sqrt{g}\right)\left(\frac{F^{2}}{2}\right)_{q}-\left(\frac{\partial}{\partial q} \log \sqrt{g}\right)\left(\frac{F^{2}}{2}\right)_{p}\right) \\
& =\frac{3\left(1-\epsilon^{2}\right) p q}{[\Delta(p, q)]^{3 / 2}}\left(p^{4}-q^{4}\right),
\end{aligned}
$$

where $\left\{e_{1}, e_{2}\right\}$ is the Berwald frame with $\omega\left(e_{1}\right)=0$. Let $V$ denote a vector field on $M$ defined by

$$
V:=\frac{\partial}{\partial t}
$$

By using the isomorphism $T_{x} M \simeq \mathbb{R}^{2}$, we have $F\left(x, V_{x}\right)<1$ on $M$. Denote the lift of $V$ by $X_{V}$. Then

$$
X_{V}=V+y^{j} \frac{\partial V^{i}}{\partial x^{j}} \frac{\partial}{\partial y^{i}}=V
$$

[Huang and Mo 2011]. It follows that

$$
X_{V}(F)=\frac{\partial F}{\partial t}=-\frac{1}{t} F,
$$

where we have made use of (5-1). Thus $V$ is conformal with dilation $c=-1 /(2 t)$ (see [Huang and Mo 2013, Lemma 3.1]). In particular, $V$ is not homothetic.

Now we calculate the following scalar function on $S M$.

$$
\xi(x, y):=\frac{A_{(x,[y])}(u, \nabla c, u)}{h_{(x,[y])}(u, u)},
$$

where $u \wedge y \neq 0$. Taking $u=e_{1}$ we obtain

$$
h_{(x,[y])}\left(e_{1}, e_{1}\right)=1, \quad A_{(x,[y])}\left(e_{1}, e_{1}, e_{1}\right)=I(x, y), \quad A_{(x,[y])}\left(e_{1}, e_{2}, e_{1}\right)=0 .
$$

Define $\nabla c$ by

$$
\nabla c=\lambda e_{1}+\mu e_{2},
$$

where $\left\{e_{1}, e_{2}\right\}$ is the Berwald frame on $M$. Then

$$
\begin{aligned}
\lambda(x, y) & =\boldsymbol{g}_{(x,[y])}\left(\nabla c, e_{1}\right) \\
& =\frac{\partial c}{\partial s}\left(-\frac{F_{q}}{\sqrt{g}}\right)+\frac{\partial c}{\partial t} \frac{F_{p}}{\sqrt{g}}=\frac{p\left(p^{2}+\epsilon q^{2}\right)}{2 F \sqrt{\Delta(p, q)} t^{2}},
\end{aligned}
$$


where $\boldsymbol{g}$ denotes the fundamental tensor. From (5-10), (5-11) and (5-12), it follows that

$$
\begin{aligned}
\xi(x, y) & =\frac{A_{(x,[y])}\left(e_{1}, \lambda e_{1}+\mu e_{2}, e_{1}\right)}{h_{(x,[y])}\left(e_{1}, e_{1}\right)} \\
& =\lambda(x, y) A_{(x,[y])}\left(e_{1}, e_{1}, e_{1}\right)=\lambda(x, y) I(x, y),
\end{aligned}
$$

where $\lambda$ and $I$ are given in (5-13) and (5-8) respectively.

Now we consider the navigation data $(F, V)$, where $F$ and $V$ are defined in (5-1) and (5-9) respectively. $(F, V)$ produces a new Finsler metric $\tilde{F}$ by

$$
F\left(x, \frac{y}{\widetilde{F}(x, y)}+V_{x}\right)=1, \quad \forall x \in M, y \in T_{x} M .
$$

By (5-1), (5-2) and (5-9), (5-14) holds if and only if

$$
p^{4}+2 \epsilon p^{2}(q+\widetilde{F})^{2}+(q+\widetilde{F})^{4}=t^{4} \widetilde{F}^{4},
$$

that is, $\widetilde{F}$ is the unique nonnegative solution of (5-15). By direct calculation we have

$$
\frac{y^{i} c_{x^{i}}}{\widetilde{F}(x, y)}=\frac{q}{2 t^{2} \widetilde{F}(x, y)}, \quad-c^{2}+2 V(c)=\frac{3}{4 t^{2}} .
$$

For the Finsler surface $(M, F), F$ is of scalar flag curvature. Using Theorem 1.1, we obtain that the Gaussian curvature $K_{\widetilde{F}}$ is given by

$$
\begin{aligned}
K_{\widetilde{F}}(x, y) & =K_{F}(x, \tilde{y})+\left[3 \frac{y^{i} c_{x^{i}}}{\widetilde{F}(x, y)}-c^{2}+2 V(c)\right]-2 \frac{A_{(x,[\tilde{y}])}(u, \nabla c, u)}{h_{(x, \tilde{y}])}(u, u)} \\
& =K_{F}(x, \tilde{y})+\frac{3 q}{2 t^{2} \widetilde{F}(x, y)}+\frac{3}{4 t^{2}}-2 \lambda(x, \tilde{y}) I(x, \tilde{y}),
\end{aligned}
$$

where

$$
\tilde{y}=y+F(x, y) V=\left(p, q+\frac{\left(p^{4}+2 \epsilon p^{2} q^{2}+q^{4}\right)^{1 / 4}}{t}\right)
$$

and $K_{F}, \lambda, I$ are given in (5-3), (5-13) and (5-8) respectively.

Let us take a look at the special case when $\epsilon=1$,

$$
F(s, t ; p, q):=\frac{\left(p^{2}+q^{2}\right)^{1 / 2}}{t} .
$$

$F$ is the famous Poincaré metric of constant sectional curvature $K_{F}=-1$. In this case, $\widetilde{F}$ is of Randers type and its Gaussian curvature is given by

$$
K_{\widetilde{F}}(x, y)=\frac{3}{4 t^{2}}\left(\frac{2 q}{\widetilde{F}(x, y)}+1\right)-1 .
$$




\section{References}

[Bao and Chern 1993] D. Bao and S.-S. Chern, "On a notable connection in Finsler geometry", Houston J. Math. 19:1 (1993), 135-180. MR 94g:53049 Zbl 0787.53018

[Bao et al. 2004] D. Bao, C. Robles, and Z. Shen, "Zermelo navigation on Riemannian manifolds", J. Differential Geom. 66:3 (2004), 377-435. MR 2005k:58023 Zbl 1078.53073

[Blair 2002] D. E. Blair, Riemannian geometry of contact and symplectic manifolds, Progress in Mathematics 203, Birkhäuser, Boston, 2002. MR 2002m:53120 Zbl 1011.53001

[Bryant 2002] R. L. Bryant, "Some remarks on Finsler manifolds with constant flag curvature", Houston J. Math. 28:2 (2002), 221-262. MR 2003h:53102 Zbl 1027.53086

[Chen et al. 2003] X. Chen, X. Mo, and Z. Shen, "On the flag curvature of Finsler metrics of scalar curvature", J. London Math. Soc. (2) 68:3 (2003), 762-780. MR 2004h:53103 Zbl 1063.53078

[Cheng and Shen 2009] X. Cheng and Z. Shen, "Randers metrics of scalar flag curvature", J. Aust. Math. Soc. 87:3 (2009), 359-370. MR 2011e:53121 Zbl 1182.53022

[Chern 1996] S.-S. Chern, "Riemannian geometry as a special case of Finsler geometry", pp. 51-58 in Finsler geometry (Seattle, WA, 1995), edited by D. Bao et al., Contemp. Math. 196, Amer. Math. Soc., Providence, RI, 1996. MR 98e:53026 Zbl 0868.53051

[Chern and Shen 2005] S.-S. Chern and Z. Shen, Riemann-Finsler geometry, Nankai Tracts in Mathematics 6, World Scientific, Hackensack, NJ, 2005. MR 2006d:53094 Zbl 1085.53066

[Hu and Deng 2012] Z. Hu and S. Deng, "Homogeneous Randers spaces with isotropic S-curvature and positive flag curvature”, Math. Z. 270:3-4 (2012), 989-1009. MR 2892934 Zbl 1239.53095

[Huang and Mo 2011] L. Huang and X. Mo, "On geodesics of Finsler metrics via navigation problem", Proc. Amer. Math. Soc. 139:8 (2011), 3015-3024. MR 2012e:53141 Zbl 1261.53037

[Huang and Mo 2013] L. Huang and X. Mo, "On conformal fields of a Randers metric with isotropic S-curvature”, Illinois J. Math. 57:3 (2013), 685-696. MR 3275734 Zbl 1303.53033

[Miron et al. 2001] R. Miron, D. Hrimiuc, H. Shimada, and S. V. Sabau, The geometry of Hamilton and Lagrange spaces, Fundamental Theories of Physics 118, Kluwer, Dordrecht, 2001. MR 2002e:53111 Zbl 1001.53053

[Mo 2008] X. Mo, "A global classification result for Randers metrics of scalar curvature on closed manifolds”, Nonlinear Anal. 69:9 (2008), 2996-3004. MR 2009k:53195 Zbl 1237.53029

[Mo and Hang 2007] X. Mo and L. Hang, "On curvature decreasing property of a class of navigation problems”, Publ. Math. Debrecen 71:1-2 (2007), 141-163. MR 2008g:53092 Zbl 1136.53022

[Shen 2001] Z. Shen, Differential geometry of spray and Finsler spaces, Kluwer, Dordrecht, 2001. MR 2003k:53090 Zbl 1009.53004

[Shen 2002] Z. Shen, "Two-dimensional Finsler metrics with constant flag curvature", Manuscripta Math. 109:3 (2002), 349-366. MR 2003k:53091 Zbl 1027.53093

[Shen 2003] Z. Shen, "Finsler metrics with $\boldsymbol{K}=0$ and $\boldsymbol{S}=0$ ", Canad. J. Math. 55:1 (2003), 112-132. MR 2004e:53112 Zbl 1035.53104

[Shen 2004] Z. Shen, "Landsberg curvature, $S$-curvature and Riemann curvature", pp. 303-355 in A sampler of Riemann-Finsler geometry, edited by D. Bao et al., Math. Sci. Res. Inst. Publ. 50, Cambridge Univ. Press, 2004. MR 2005k:53132 Zbl 1074.53063

[Shen and Xia 2012] Z. Shen and Q. Xia, "On conformal vector fields on Randers manifolds", Sci. China Math. 55:9 (2012), 1869-1882. MR 2960866 Zbl 1267.53027

[Shen and Xing 2008] Z. M. Shen and H. Xing, "On Randers metrics with isotropic $S$-curvature", Acta Math. Sin. (Engl. Ser.) 24:5 (2008), 789-796. MR 2010c:53109 Zbl 1151.53065 
[Xia 2013] Q. Xia, "On the flag curvature of a class of Randers metric generated from the navigation problem”, J. Math. Anal. Appl. 397:1 (2013), 415-427. MR 2969001 Zbl 1254.53045

[Xing 2005] H. Xing, "The geometric meaning of Randers metrics with isotropic $S$-curvature", $A d v$. Math. (China) 34:6 (2005), 717-730. MR 2006m:53117

[Zermelo 1931] E. Zermelo, "Über das Navigationsproblem bei ruhender oder veränderlicher Windverteilung”, Z. Angew. Math. Mech. 11:2 (1931), 114-124. Zbl 0001.34101

Received December 15, 2013.

LIBING HUANG

SCHOOL OF MATHEMATICAL SCIENCES

NANKAI UNIVERSITY

TIANJIN, 300071

CHINA

huanglb@nankai.edu.cn

XiAOHUAN Mo

Key Laboratory of PuRe ANd ApPlied Mathematics

SCHOOL OF MATHEMATICAL SCIENCES

PEKING UNIVERSITY

BEIJING, 100871

CHINA

moxh@pku.edu.cn 


\title{
PACIFIC JOURNAL OF MATHEMATICS
}

\author{
msp.org/pjm
}

Founded in 1951 by E. F. Beckenbach (1906-1982) and F. Wolf (1904-1989)

\section{EDITORS}

Don Blasius (Managing Editor)

Department of Mathematics

University of California

Los Angeles, CA 90095-1555

blasius@math.ucla.edu

\author{
Paul Balmer \\ Department of Mathematics \\ University of California \\ Los Angeles, CA 90095-1555 \\ balmer@math.ucla.edu \\ Robert Finn \\ Department of Mathematics \\ Stanford University \\ Stanford, CA 94305-2125 \\ finn@math.stanford.edu \\ Sorin Popa \\ Department of Mathematics \\ University of California \\ Los Angeles, CA 90095-1555 \\ popa@math.ucla.edu
}

\author{
Vyjayanthi Chari \\ Department of Mathematics \\ University of California \\ Riverside, CA 92521-0135 \\ chari@math.ucr.edu \\ Kefeng Liu \\ Department of Mathematics \\ University of California \\ Los Angeles, CA 90095-1555 \\ liu@math.ucla.edu \\ Jie Qing \\ Department of Mathematics \\ University of California \\ Santa Cruz, CA 95064 \\ qing@ cats.ucsc.edu
}

\section{PRODUCTION}

Silvio Levy, Scientific Editor, production@msp.org

\section{SUPPORTING INSTITUTIONS}

ACADEMIA SINICA, TAIPEI

CALIFORNIA INST. OF TECHNOLOGY

INST. DE MATEMÁTICA PURA E APLICADA

KEIO UNIVERSITY

MATH. SCIENCES RESEARCH INSTITUTE

NEW MEXICO STATE UNIV.

OREGON STATE UNIV.

\author{
STANFORD UNIVERSITY \\ UNIV. OF BRITISH COLUMBIA \\ UNIV. OF CALIFORNIA, BERKELEY \\ UNIV. OF CALIFORNIA, DAVIS \\ UNIV. OF CALIFORNIA, LOS ANGELES \\ UNIV. OF CALIFORNIA, RIVERSIDE \\ UNIV. OF CALIFORNIA, SAN DIEGO \\ UNIV. OF CALIF., SANTA BARBARA
}

\author{
Daryl Cooper \\ Department of Mathematics \\ University of California \\ Santa Barbara, CA 93106-3080 \\ cooper@math.ucsb.edu \\ Jiang-Hua Lu \\ Department of Mathematics \\ The University of Hong Kong \\ Pokfulam Rd., Hong Kong \\ jhlu@maths.hku.hk \\ Paul Yang \\ Department of Mathematics \\ Princeton University \\ Princeton NJ 08544-1000 \\ yang@math.princeton.edu
}

These supporting institutions contribute to the cost of publication of this Journal, but they are not owners or publishers and have no responsibility for its contents or policies.

See inside back cover or msp.org/pjm for submission instructions.

The subscription price for 2015 is US \$420/year for the electronic version, and \$570/year for print and electronic.

Subscriptions, requests for back issues and changes of subscribers address should be sent to Pacific Journal of Mathematics, P.O. Box 4163, Berkeley, CA 94704-0163, U.S.A. The Pacific Journal of Mathematics is indexed by Mathematical Reviews, Zentralblatt MATH, PASCAL CNRS Index, Referativnyi Zhurnal, Current Mathematical Publications and Web of Knowledge (Science Citation Index).

The Pacific Journal of Mathematics (ISSN 0030-8730) at the University of California, c/o Department of Mathematics, 798 Evans Hall \#3840, Berkeley, CA 94720-3840, is published twelve times a year. Periodical rate postage paid at Berkeley, CA 94704, and additional mailing offices. POSTMASTER: send address changes to Pacific Journal of Mathematics, P.O. Box 4163, Berkeley, CA 94704-0163.

PJM peer review and production are managed by EditFLOW ${ }^{\circledR}$ from Mathematical Sciences Publishers.

\section{PUBLISHED BY}

\section{mathematical sciences publishers \\ nonprofit scientific publishing}

http://msp.org/

(C) 2015 Mathematical Sciences Publishers 


\section{PACIFIC JOURNAL OF MATHEMATICS}

Volume $277 \quad$ No. $1 \quad$ September 2015

Real positivity and approximate identities in Banach algebras

DAVID P. BLECHER and NARUTAKA OZAWA

On shrinking gradient Ricci solitons with nonnegative sectional curvature 61

Mingliang CAI

From quasimodes to resonances: exponentially decaying perturbations

ORAN GANNOT

A general simple relative trace formula

JAYCE R. GETZ and HEEKYOUNG HAHN

Chern-Simons functions on toric Calabi-Yau threefolds and

Donaldson-Thomas theory

\section{ZHENG HUA}

On the flag curvature of a class of Finsler metrics produced by the navigation problem

LiBING HUANG and XIAOHUAN MO

Angular distribution of diameters for spheres and rays for planes

NOBUHIRO INNAMI and YUYA UNEME

A note on an $L^{p}$-Brunn-Minkowski inequality for convex measures in the 187 unconditional case

ARNAUD MARSIGLIETTI

Structure of seeds in generalized cluster algebras

TOMOKI NAKANISHI

Inequalities of Alexandrov-Fenchel type for convex hypersurfaces in hyperbolic space and in the sphere

Yong WeI and ChangWei XiONG

Upper bounds of root discriminant lower bounds 